\title{
Resilient Critical Infrastructure Planning Under Disruptions Considering Recovery Scheduling
}

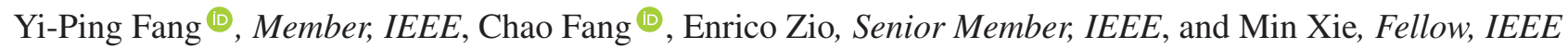

\begin{abstract}
Reliable and safe critical infrastructures are crucial for the sustainability of modern societies. To cope with increasing disruptive events such as man-made and natural disasters attacking infrastructures, resilience should be considered as an integrated perspective into the system planning process. This paper presents a $p$-robust optimization model for infrastructure network planning against spatially localized disruptions. The optimization aims at minimizing the investment costs for system hardening and expansion and the total system costs under nominal operating conditions, while incorporating resilience requirements by the $p$-robustness constraints. Importantly, instead of only mitigating system vulnerability, the proposed model integrates the arranging of the repair sequence of damaged components under limited repair resources into the preevent system planning. The complexity of the proposed model is analyzed, and a hybrid algorithm that combines scenariobased decomposition and variable neighborhood search is developed for its efficient solution. The effectiveness of the approach is illustrated through an application to a real power transmission system. Quantitative analysis can assist managers in decision making regarding investing in different system protection actions and making a tradeoff between desired resilience and budget constraints.
\end{abstract}

Index Terms-Critical infrastructures (CIs), disruption risk, optimization, system planning, system resilience.

\section{NOMENCLATURE}

Indexes, parameters, and sets

$\mathbb{G}\left(V, L^{0}\right) \quad$ An undirected graph $\{\mathbb{G}\}$ comprising a set of nodes $V$ connected by a set of links $L^{0}$ representing the original network system before the disruption.

Manuscript received May 19, 2018; revised September 26, 2018 and January 28, 2019; accepted February 26, 2019. Date of publication; date of current version. This work was supported in part by the National Natural Science Foundation of China under Grant 71301123, Grant 71871171, Grant 71725007, and Grant 71673210, and in part by the Fundamental Research Funds for the Central Universities. Review of this manuscript was arranged by Department Editor Y. H. Kwak. (Corresponding author: Chao Fang.)

Y.-P. Fang is with the Chair on Systems Science and the Energy Challenge, Fondation Electricité de France, Laboratoire Génie Industriel, CentraleSupélec, Université Paris-Saclay, 91190 Gif-sur-Yvette, France (e-mail: yiping.fang@centralesupelec.fr).

C. Fang is with the School of Economics and Management, Wuhan University, Wuhan 430072, China (e-mail: chao.fang@whu.edu.cn).

E. Zio is with the Centre for Research on Risk and Crisis, Mines ParisTech, PSL Research University, F-06904 Sophia Antipolis, France, and also with the Department of Energy, Politecnico di Milano, 20156 Milano, Italy (e-mail: enrico.zio@polimi.it).

M. Xie is with the Department of Systems Engineering and Engineering Management, City University of Hong Kong, Hong Kong (e-mail: minxie@cityu.edu.hk).

Color versions of one or more of the figures in this paper are available online at http://ieeexplore.ieee.org.

Digital Object Identifier 10.1109/TEM.2019.2902916
$V_{G}, V_{T}, V_{D}$

$S$

$L^{\prime}$

$L$

$n$

l

$i$

$t$

$s$

Parameters

$B$

$T_{s}$

$\bar{f}_{l}$

$\bar{g}_{n}$

$\bar{p}_{n}$

$o(l)$

$d(l)$

K

$\rho_{i}$

$a_{i s}$

$c_{i}^{\text {har }}$

$c_{l}^{\text {inv }}$

$c_{n}^{\mathrm{o} \& \mathrm{~m}}$

$c_{n}^{\mathrm{emi}}$

$c_{l}^{\mathrm{o} \& \mathrm{~m}}$

$c_{n}^{\mathrm{e} \& \mathrm{~s}}$

$C_{\text {INV }} \quad$ Annualized investment costs [dollar].

$C_{\mathrm{OPC}} \quad$ Total (hourly) system costs under nominal operation condition [dollar].

Sets of supply, transship, and demand nodes, respectively, $V_{G} \cup V_{T} \cup V_{D}=V$.

Set of all SLD scenarios considered.

Set of prospective transmission lines for expansion.

Union of sets $L^{0}$ and $L^{\prime}$.

Index of nodes.

Index of lines.

Index of nodes and lines, $i \in\{n, l\}$.

Index of time period.

Index of disruption scenario.

Total budget available for building new lines and hardening existing facilities [dollar].

Total time periods of system restoration under scenario $s \in S$.

Flow capacity of line $l \in L=L^{0} \cup L^{\prime}$.

Supply capacity in supply node $n \in V_{G}$.

Expected demand at node $n \in V_{D}$.

Origin or sending node of line $l$.

Destination or receiving node of line $l$.

Number of repair crews available for postdisruption system restoration activity.

Time duration required to repair a damaged facility $i \in\{n, l\}$, e.g., [hour].

$a_{i s}=0$ if facility $i$ is damaged in scenario $s \in S$, 1 otherwise.

Annualized cost of investment in hardening facility $i$ [dollar].

Annualized cost of investment in constructing/expanding transmission line $l \in L^{\prime}$ [dollar].

O\&M cost associated with unit of flow generated at node $n \in V_{G}$ [dollar per unit of flow].

Emission (environmental) cost associated with unit of flow generated at node $n \in V_{G}$, [dollar per unit of flow].

Operational and maintenance cost associated with unit of flow through arc $l \in L$ [dollar per unit of flow].

Economic and societal cost of each unit of interrupted service at demand node $n \in V_{D}$ [dollar per unit of flow]. 
$\sigma \quad$ Weighting parameter making $C_{\mathrm{OPC}}$ and $C_{\mathrm{INV}}$ the same scale.

$C_{s}^{*} \quad$ Optimum scenario penalty costs [dollar].

$p \quad$ A given constant setting the upper bounds on the maximal allowable relative regret for each disruption scenario.

\section{Decision variables}

$v_{l} \quad v_{l}=1$ if prospective line $l \in L^{\prime}$ is built, 0 otherwise.

$w_{i} \quad w_{i}=1$ if a facility $i \in\{n, l\}$ is selected to be hardened, 0 otherwise

$r_{i t s} \quad r_{i t s}=1$ if facility $i$ is repaired at the beginning of time $t$ under scenario $s$, otherwise 0 .

Auxiliary decision variables

$x_{i t s} \quad x_{i t s}=1$ if facility $i$ is functional at the beginning

of time period $t$ under scenario $s$, otherwise 0 .

$f_{l t s} \quad$ Amount of flow passing through arc $l \in L$ at time $t$ under scenario $s$.

$g_{n t s} \quad$ Amount of generated flow at node $n \in V_{G}$ at time $t$ under scenario $s$.

$\Delta p_{n t s} \quad$ Amount of unsatisfied demand at node $n \in V_{D}$ at time $t$ under scenario $s$.

$f_{l}, g_{n}, \Delta p_{n} \quad$ Variables under the nominal operation scenario, i.e., the scenario without disruption, corresponding to $f_{l t s}, g_{n t s}, \Delta p_{n t s}$ that are used for disruptive scenario $s$.

\section{INTRODUCTION}

C RITICAL infrastructure (CIs) systems (such as energy, transportation, water distribution, and other lifeline networks) are indispensable for modern societies to provide essential services that support economic prosperity, social security and stability, and quality of life [1]-[4]. Nowadays, these infrastructure systems suffer increasing disruption risks in terms of both frequency and consequences, e.g., man-made or natural disasters and terrorist attacks, making critical infrastructure protection (CIP) a priority for our societies [4]. To strengthen the security and resilience of national/regional CIs, many governments and organizations have initiated CIP plans, in which the concept of infrastructure "resilience" has been highlighted [5].

The term "resilience" has many definitions, without a broadly accepted one, even only focusing on CIs [6], [7]. A complicating aspect in previous attempts to define resilience is the recognition that "resilience is a family of related ideas, not a single thing" [8]. Following our previous studies [7], [9], [10] and being consistent with most of the literature [6], we define resilience of a CI system as the joint ability of this system to resist (prevent and withstand) any possible hazard, absorb initial damage, and quickly recover to normal operation. It can be quantified based upon the system real functionality curve under disruptions and the targeted functionality curve without disruption during a recovery time horizon.

In the case of failure due to disruptions (e.g., technical accidents or intentional attacks), preevent actions and postevent solutions are needed to mitigate disruptive damages and recover the system to normal conditions. Identification and optimization of preevent measures for protecting infrastructures has been a focus problem in the related works [4], [11]-[13]. Preevent measures proposed in the literature include risk screening [14], enhancing system redundancy [15], [16], hardening system components [7], [17]-[19], optimizing system design [20], [21], etc. However, most of the studies on the optimization of preevent measures do not consider the postevent restoration processes and only take the performance drop or system operation cost functions immediately after the disruptions (i.e., robustness) as the objective function (robustness is indeed one of the components of system resilience) [22]. For the postevent restoration, many quantitative restoration models have been proposed for investigating the community restoration time [23], [24], evaluating the direct and indirect losses of CI systems under disruptions [25], [26], studying the effect of interdependencies on postevent CI functionalities [27]. Also, there have been some studies on postevent CI restoration planning, aimed at identifying the optimum restoration operations at the infrastructure component level, e.g., scheduling the repair sequence of damaged components and allocating limited repair resources, for a rapid system performance recovery [10], [28]-[31]. However, very few investigations integrate postevent recovery into the preevent planning and management of networked CIs. Some studies considered the recovery process, but they did not consider the restoration sequence of damaged components [32]-[35].

To bridge this gap, this paper introduces a mixed-integer programming (MIP) model to optimize the $p$-robustness CI expansion and protection planning ( $p$-CIEPP) against spatially localized disruptions (SLDs). In particular, the model seeks the minimum-nominal-cost solution that is $p$-robust, i.e., whose relative regret is no more than $100 p \%$ in each SLD scenario (higher value of $p$ means that the decision maker (DM) can tolerate a greater loss under each disruption scenario). Instead of only mitigating system vulnerability, the proposed model for the first time integrates the arranging of the repair sequence of damaged components under limited repair resources into the preevent system planning. As considering the restoration process makes the model very difficult to be solved, a hybrid algorithm that combines scenario-based decomposition (SBD) with variable neighborhood search (VNS) [36] is proposed to efficiently solve the problem. By applying the proposed approach to a case study of real power transmission systems, it shows the ability to support decision making such as how to better invest in system hardening and protection for resilient CIs.

The model proposed in this paper aims to find expansion and protection plans for CIs that make the systems perform well under nominal conditions and, at the same time, is able to efficiently deal with short-term disruptions. The $p$-robustness measure is adopted mainly because of the following reasons: 1$)$ the optimization with the $p$-robustness measure is flexible enough to accommodate other objectives, such as the nominal investment and operation costs, considered in this study, while other robust criteria like minimax cost and minimax regret [37] cannot easily do so; and 2) the proposed model is motivated by the needs of 
real CI DMs, e.g., power system utilities, who usually wish to limit the regret in each possible contingency to bound system exposure to risk, but are even more concerned with minimizing the nominal operation cost, knowing that the nominal scenario is the most likely to occur and that it is typically difficult to estimate the probabilities of disruption scenarios in practice.

The contributions of this paper are highlighted as follows.

1) A quantitative optimization model with the $p$-robustness measure that integrates system resilience is proposed for the planning and management of networked CIs.

2) The model integrates the arranging of the repair sequence of damaged components under limited repair resources into the preevent system planning.

3) The complexity of the proposed model is rigorously analyzed.

4) An efficient hybrid algorithm that combines SBD with VNS is proposed for the solution of the problem.

The rest of this paper is organized as follows. Section II introduces a $p$-robustness optimization model and analyzes the problem complexity. Section III develops a hybrid SBD-VNS algorithm to solve the model. Section IV illustrates the application of the approach to a real power transmission system and discusses managerial implications based on the computational results. Finally, Section V concludes this paper.

\section{PROBlem DescriPtion AND MODEL Formulation}

In this paper, we study the CI expansion and protection planning (CIEPP) problem by taking into account the resilience of the system. The problem is addressed from the perspective of a centralized decision-making agency, which pursues the overall welfare of the system, e.g., a public transmission system operator of power systems. In particular, a $p$-robustness mathematical model is proposed with the objective of minimizing the total cost, which is the sum of the investment costs and the total system costs under nominal operating condition. Meanwhile, the system is protected against potential disruptive events by enforcing an acceptable regret level of system resilience loss for all the disruption scenarios considered. In other words, system resilience is represented by the $p$-robustness constraint. The decisions of the problem are made in two stages: planning decisions and recourse actions. In the first stage, two user-defined system investment/protection options are considered: 1) hardening existing facilities (nodes and lines) to lower the probability of damage; and 2) building new lines to add system redundancy. The planning decisions are determined before the realization of the uncertain disruptive events is known. Once a disruptive event is observed, recourse actions, including allocating repair resources, scheduling repair tasks, and reassigning network flows, may be deployed, which are regarded as the second-stage decisions.

The assumptions of the CIEPP model referring to the flows and capacities of CI systems, the disruptions studied, and the costs related to the investment and operation of the system, are listed as follows.

1) The CI network is composed of links and nodes serving for transporting commodities/energy/information, etc., which can be represented by network flows.

2) There is a known demand or supply for each node and a known flow capacity for each link.
3) Links and nodes are subject to failure, and failed ones are completely unusable before being repaired or reconstructed.

4) The time duration used to repair each damaged component is given, and there are a known number of repair crews for system restoration.

5) The DMs have a budget to invest in protecting existing nodes/links or constructing new ones so that they are invulnerable to disruptive scenarios.

\section{A. Problem Statement}

A networked CI system, such as a power grid, a water distribution network, or a communication network, is modeled by an undirected network $\mathbb{G}\left(V, L^{0}\right)$. Each node in such a system $n \in V$ might represent generators, buses for electric power systems, or pump stations, and compressors for pipeline systems, and each link $l \in L^{0}$ could be transmission lines, transformers for electric power systems, or gas pipeline segments for pipeline systems. Let $f_{l}$ be the amount of energy, data, or load, etc., transferred along with line $l$. There is a set of supply or source nodes in the system, $V_{G} \subseteq V$, a set of transshipment nodes, $V_{T} \subseteq V$, and a set of demand nodes, $V_{D} \subseteq V$. Each line $l \in L^{0}$ has an associated flow capacity $\bar{f}_{l} \in \mathbb{R}_{+}$, while each supply node $n \in V_{G}$ has a supply capacity $\bar{g}_{n} \in \mathbb{R}_{+}$, and each demand node $n \in V_{D}$ has a demand $\bar{p}_{n} \in \mathbb{R}_{+}$for its nominal operation.

As mentioned before, two user-defined system protection practices are considered in the planning of system expansion and protection. First, we assume that the DMs have the ability to protect nodes or lines so that they are invulnerable to damage, which means that the protected facilities can still work even after a disruptive event occurs. Second, the DMs are willing to build any new lines $L^{\prime}$, and any newly built line is invulnerable to disruption (i.e., protection and hardening measures will be adopted for all the newly constructed lines). The union of $L^{0}$ and $L^{\prime}$ is denoted as $L$. Indeed, it is essentially difficult or impossible to protect CI system components to make them invulnerable to any disruption, although grid-hardening measures like undergrounding power lines and upgrading transmission line materials (e.g., high-temperature and low-sag conductors) in power systems can make the lines reasonably invulnerable to certain disruptive scenarios, such as high winds and extreme heat. In this paper, the ultimate goal is to identify system components, which, when "hardened," yield the best improvement in system resilience. Therefore, for simplicity and not considering extreme severe events, we adopt the common assumption that the DMs have the capacity to protect vulnerable components so that they can survive and keep operating during a disruptive event [7], [17], [38], [39]. Fig. 1(a) presents a simple infrastructure network with two protection measures.

Then, we let binary variables $v_{l}$ and $w_{i}$ represent the decisions of building prospective lines $l \in L^{\prime}$ and hardening existing facilities $i \in V \cup L^{0}$, and their annualized investment costs are denoted by $c_{l}^{\text {inv }}$ and $c_{i}^{\text {har }}$, respectively. Hence, the annualized investment cost is represented as

$$
C_{\mathrm{INV}}=\sum_{i \in V \cup L^{0}} c_{i}^{\mathrm{har}} w_{i}+\sum_{l \in L^{\prime}} c_{l}^{\mathrm{inv}} v_{l}
$$




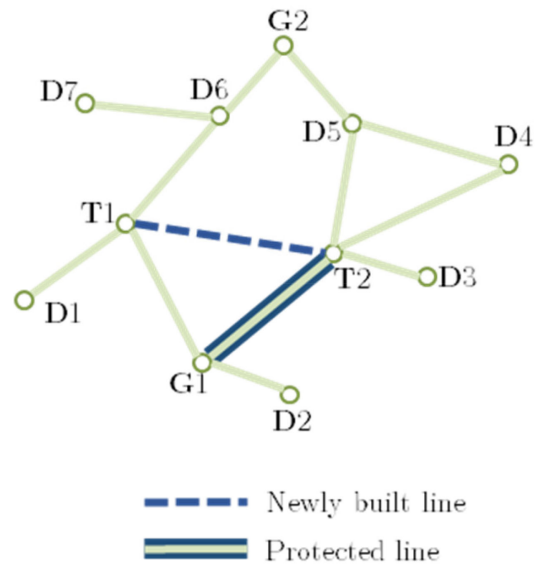

(a)

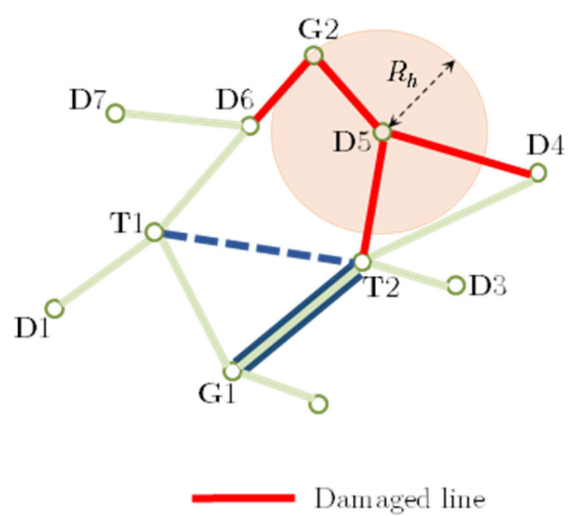

(b)

Fig. 1. (a) Notional infrastructure system: circles labeled with $\mathrm{G}$ represent the source nodes, circles labeled with $\mathrm{T}$ represent transshipment nodes, and circles labeled with D represent the demand nodes. (b) Illustrative SLD scenario whose epicenter is located at D5 and with an influence radius of $R_{h}$.

The definition of investment costs in (1) can be easily modified by adding (removing) the related costs if more (fewer) protection measures are considered for specific CI systems.

In addition, there is an operational and maintenance $(\mathrm{O} \& \mathrm{M})$ $\operatorname{cost} c_{n}^{\mathrm{o} \& \mathrm{~m}}$ and an emission $\operatorname{cost} c_{n}^{\mathrm{emi}}$ associated with a unit of flow generation and an O\&M cost $c_{l}^{\text {o\&m }}$ associated with a unit of flow through arc $l \in L$. Furthermore, the impact of the interruption of service at each demand node on the community is determined by the amount of the interrupted demand multiplied by a penalty $\operatorname{cost} c_{n}^{\text {e\&s }}$, which represents the average economic and societal value of the interrupted demand, and is usually measured in monetary units. For example, in power systems, the penalty cost of lost load can be calculated using the commonly used value of lost load [40], [41], which is defined as the estimated amount that power customers receiving electricity with firm contracts would be willing to pay to avoid a disruption in their electricity service [40]. Similar definitions can be applied when other CI systems are considered, e.g., the cost of disruption of gas supply [42] and the cost of welfare losses from urban water supply disruptions [43]. Accordingly, the total system costs under nominal operation condition can be represented as

$$
\begin{aligned}
C_{\mathrm{OPC}}= & \sum_{n \in V_{G}}\left(c_{n}^{\mathrm{o \& m}}+c_{n}^{\mathrm{emi}}\right) g_{n} \\
& +\sum_{l \in L} c_{l}^{\mathrm{o \& m}} f_{l}+\sum_{n \in V_{D}} c_{n}^{\mathrm{e} \& \mathrm{~s}} \Delta p_{n}
\end{aligned}
$$

where $g_{n}$ is the amount of generated flow at node $n \in V_{G}$ within a unit of time (e.g., $1 \mathrm{~h}$ ), and $\Delta p_{n}$ is the unsatisfied service at the demand node $n \in V_{D}$. The total system costs under nominal operation conditions $C_{\mathrm{OPC}}$ comprise: 1) the O\&M costs and emission costs for generating unit at all suppliers; 2) the O\&M costs for transporting unit across the whole network; and 3 ) the economic penalty costs for all unmet services at the demand nodes. It is noted that this definition of system costs by (2) is inspired by the operational costs of electricity infrastructures, including the power generation costs (first item), power transmission costs (second item), and lost load costs (third item).
When applying (2) to other CIs like water distribution networks, some costs may not exist, e.g., the emission costs; then, the corresponding coefficients can simply take the value of zero.

Consequently, the objective of the CIEPP is to minimize the sum of the annualized investment costs $C_{\mathrm{INV}}$ and the total system costs under nominal operation condition $C_{\mathrm{OPC}}$

$$
\min C_{\mathrm{INV}}+\sigma \cdot C_{\mathrm{OPC}}
$$

where $\sigma$ is a weighting/discount factor to make investment and operation costs comparable. Note that the objective function (3) is general enough for the CIEPP problem, though the definitions of $C_{\mathrm{INV}}$ and $C_{\mathrm{OPC}}$ may vary when applying to different $\mathrm{CI}$ systems.

Another objective of the CIEPP is system resilience, for which we consider a set of scenarios representing potential disruptive events that may occur on the CI system, denoted by $S$, and each of the scenarios specifies a set of facilities that are disrupted simultaneously. An attacked facility will become nonoperational, i.e., unable to transmit network flow, if it has not been protected. Without loss of generality, any unrepaired facility is assumed to lose its function during the considered time horizon. The functionality of system facilities is modeled here by binary states, i.e., fully operational and nonoperational.

In this paper, we integrate the arranging of the repair sequence of damaged components under limited repair resources into the preevent CIEPP framework. We assume that the time duration used to repair each damaged facility is given, and there is a known number of repair crews, which are the only limited resources for system restoration. In practice, there are other limited resources like the spare parts for CI system restoration. However, it is difficult or even impossible to model all the required repair resources if we are not looking at the details of component types and their damage types/levels. Therefore, in the literature, the number of repair crews is usually considered as the only limited resource for general infrastructure systems [7], [41]. Actually, repair crews here are not necessarily only the repair workers: it can be understood as a group of repair workers with adequate material resources required to carry out the repair tasks. 
Formally, let $T_{s}$ be the total time periods of system restoration under disruptive scenario $s \in S$, and $\Delta p_{n t s}$ be the amount of unsatisfied demand at node $n \in V_{D}$ at time $t \in\left\{1 \ldots T_{s}\right\}$ under scenario $s \in S$; then, the penalty cost for system performance loss under scenario $s$ (or called scenario cost) is given by

$$
C_{\text {RESI }}(s)=\sum_{n \in V_{D}} c_{n}^{\mathrm{e} \& s} \sum_{t=1}^{T_{s}} \Delta p_{n t s} .
$$

Note that $C_{\text {RESI }}(s)$ takes into account the cumulative system functionality loss over the whole restoration horizon, representing the loss of system resilience under disruption scenario $s$. This definition of resilience loss is equivalent to the size of the so-called resilience triangle [22]. Then, the goal is to ensure that the scenario cost $C_{\mathrm{RESI}}(s)$ is within an acceptable boundary for all the considered scenarios in order to achieve system resilience. This objective is implemented in this paper by the $p$-robust criterion [44], [45], which will be introduced in detail in the following section.

\section{B. p-CIEPP Model}

A $p$-robustness optimization formulation is provided in this section for the CIEPP problem described in Section II-A. The objective of the $p$-CIEPP is given by

$$
\min C_{\mathrm{INV}}+\sigma \Delta C_{\mathrm{OPC}}
$$

which seeks to minimize the sum of the annualized investment $\operatorname{costs} C_{\mathrm{INV}}$ and the weighted total system costs under nominal operation condition $C_{\mathrm{OPC}}$. This objective function quantifies the total nominal costs of a CI system in terms of its economic, environmental, and societal impacts. We assume that the latter two can be transformed into monetary units in this paper.

The constraints of the $p$-CIEPP model are given and explained as follows.

\section{1) p-Robustness Constraints:}

$$
C_{\mathrm{RESI}}(s) \leq(1+p) C_{s}^{*} \quad \forall s \in S .
$$

Constraints (6) enforce the $p$-robust criterion [44], [45] for the system resilience loss against each disruption scenario. It requires the scenario penalty costs $C_{\mathrm{RESI}}(s)$ be not more than $100(1+p) \%$ of the optimal scenario penalty costs $C_{s}^{*}$, which is calculated by solving the CIEPP for each of the given scenario $s$, denoted as CIEPP $_{S}$ (the detailed formulation of CIEPP $_{S}$ is given at the end of this section). Note that CIEPP $_{S}$ is a deterministic (i.e., single-scenario) minimization problem, indexed by the scenario index $s$, that is, for each $s \in S$, there is a different problem CIEPP $_{S}$. The structure of these problems is identical; only the data are different. For each $s$, let $C_{s}^{*}$ be the optimal objective value for CIEPP $_{\mathrm{S}}$. The constraints (6) can be equivalently represented as

$$
\frac{C_{\mathrm{RESI}}(s)-C_{s}^{*}}{C_{s}^{*}} \leq p \quad \forall s \in S
$$

whose left-hand side is the relative regret for scenario $s$; the absolute regret is given by $C_{\mathrm{RESI}}(s)-C_{s}^{*}$. The $p$-robust measure sets upper bounds on the maximum allowable relative regret about the system resilience loss for each scenario. This definition of $p$-robustness is consistent with that given by [44]-[46].

2) Operational Constraints Under Disruptive Scenarios:

$$
\begin{aligned}
& \sum_{l \in L \mid o(l)=n} f_{l t s}-\sum_{l \in L \mid d(l)=n} f_{l t s}=g_{n t s} \quad \forall n \in V_{G} \forall t, s \\
& \sum_{l \in L \mid o(l)=n} f_{l t s}-\sum_{l \in L \mid d(l)=n} f_{l t s}=0 \quad \forall n \in V_{T} \forall t, s \\
& \sum_{l \in L \mid d(l)=n} f_{l t s}-\sum_{l \in L \mid o(l)=n} f_{l t s}+\Delta p_{n t s}=\bar{p}_{n} \\
& \quad \forall n \in V_{D} \forall t, s \\
& 0 \leq g_{n t s} \leq \bar{g}_{n} \quad \forall n \in V_{G} \forall t, s \\
& 0 \leq p_{n t s} \leq \bar{p}_{n} \quad \forall n \in V_{D} \forall t, s \\
& -x_{o(l) t s} \bar{f}_{l} \leq f_{l t s} \leq x_{o(l) t s} \bar{f}_{l} \quad \forall l \in L \forall t, s \\
& -x_{d(l) t s} \bar{f}_{l} \leq f_{l t s} \leq x_{d(l) t s} \bar{f}_{l} \quad \forall l \in L \forall t, s \\
& -x_{l t s} \bar{f}_{l} \leq f_{l t s} \leq x_{l t s} \bar{f}_{l} \quad \forall l \in L \forall t, s .
\end{aligned}
$$

Constraints (8)-(15) represent system operational restrictions under disruptive scenarios. Specifically, constraints (8)-(12) are the flow conservation constraints: for supply nodes, we require the flow out to be less than or equal to the supply capacity (8), (11); for demand nodes, we require the flow in to be equal to the nominal demand minus unmet demand (10), (12); and for transshipment nodes, we require the flow in to be equal to the flow out (9). Constraints (13)-(15) force the arc flows to fall within a given capacity taking into account that positive flows can only be achieved if the given arc, as well as its tail and head nodes, is fully functional under disruption scenario $s$.

3) Constraints Concerning System Restoration Decisions:

$$
\begin{aligned}
& x_{i t s} \geq w_{i} \quad \forall i \in L^{0} \cup V \forall t, s \\
& x_{i t s} \geq a_{i s} \quad \forall i \in L^{0} \cup V \forall t, s \\
& x_{i t s} \leq w_{i}+a_{i s}+\sum_{\tau=1}^{t-1} r_{i \tau s} \quad \forall i \in L^{0} \cup V \forall t, s \\
& x_{l t s}=v_{l} \quad \forall l \in L^{\prime} \forall t, s \\
& \sum_{t=1}^{\rho_{i}} r_{i t s}=0 \quad \forall i \in L^{0} \cup V \forall s \in S \\
& \sum_{t=1}^{T_{s}} r_{i t s} \leq 1 \quad \forall i \in L^{0} \cup V \forall s \in S \\
& \sum_{n \in V} \sum_{\tau=\min \left\{t+\rho_{n}-1, T_{s}\right\}} r_{n \tau s} \\
& +\sum_{l \in L^{0}} \sum_{\tau=t}^{\tau=\min \left\{t+\rho_{l}-1, T_{s}\right\}} r_{l \tau s} \leq K \quad \forall t, s .
\end{aligned}
$$

Formulas (16)-(22) are constraints concerning system restoration decisions, and they capture the relationships among the restoration scheduling decisions under disruption scenario $s$. 
In particular, constraints (16)-(18) ensure that a facility $i$ is out of functionality in the network at the beginning of time period $t$, if and only if it has not been hardened, has been damaged in the scenario, and has not been repaired by some repair crews before time period $t$. If facility $i$ has either been hardened, i.e., $w_{i}=1$, or not failed, i.e., $a_{i s}=1$, it will keep functioning $x_{\text {its }}=1 \forall t, s$, enforced by constraints (16) and (17); on the contrary, when $w_{i}=0$ and $a_{i s}=0$, the state of facility $i$ (i.e., $x_{\text {its }}$ ) is determined by whether it has been repaired before time $t$, i.e., the item $\sum_{\tau=1}^{t-1} r_{i \tau s}$ in constraints (18). If it has not been repaired before time $t$, i.e., $\sum_{\tau=1}^{t-1} r_{i \tau s}=0$, the right-hand side of constraints (18) is 0 , forcing $x_{\text {its }}=0$; otherwise, the right-hand side of constraints (18) is 1 , and $x_{\text {its }}$ will take the value of 1 in the costminimization problem. Constraints (19) ensure that the newly built lines are invulnerable and always operational. Constraints (20) state that a facility cannot be repaired in a period earlier than its required processing time. Constraints (21) state that a failed facility can only be repaired once at most. Constraints (22) limit the deployment of a given number of repair crews in each period. The upper bound $\tau=\min \left\{t+\rho_{l}-1, T_{s}\right\}$ is given by the smaller value between the period that the restoration of line $l$ is finished and the total time horizon of system restoration under each scenario $T_{s}$. Basically, the summation of the decision variable $r_{l \tau s}$ over the restoration time, $\sum_{\tau=t}^{\tau=\min }\left\{t+\rho_{l}-1, T_{s}\right\} r_{l \tau s}$, indicates whether one repair crew has been occupied by the restoration activity of line $l$ or not, since only when a repair crew has already been assigned to restore line $l$ at time $t$, it is possible to finish this restoration at any time $\tau \in\left[t, t+\rho_{l}-1\right]$, i.e., $r_{l \tau s}=1$ for $\tau \in\left[t, t+\rho_{l}-1\right]$.

Note that constraints (16)-(22) together with the $p$-robust criterion (6) model the postevent restoration planning, aiming to allocate and schedule limited repair crews to restore failed components so as to mitigate the system resilience loss. The system is able to recover to at most the same state as prior to the disruption when all the failed components are repaired.

4) Operational Constraints Under a Normal Operation Scenario:

$$
\begin{aligned}
& \text { (8)-(12) suppressing indices } t \text { and } s \\
& \quad-\bar{f}_{l} \leq f_{l} \leq \bar{f}_{l} \quad \forall l \in L
\end{aligned}
$$

Additionally, constraints (23) and (24) correspond to the flow conservation and line capacity constraints under a nominal operation scenario, i.e., the scenario without disruption.

5) Nature of Binary Decision Variables:

$$
\begin{aligned}
& v_{l}, w_{i} \in\{0,1\} \quad \forall l \in L^{\prime} \forall i \in L^{0} \cup V \\
& r_{i t s}, x_{i t s} \in\{0,1\} \quad \forall i \in L^{0} \cup V \forall t, s .
\end{aligned}
$$

Finally, (25) and (26) are standard integrality constraints.

The optimal scenario penalty costs $C_{s}^{*}$ in (7) are calculated by solving the CIEPP $\left(\mathrm{CIEPP}_{\mathrm{S}}\right)$ below for each of the given scenario $s$ :

$$
C_{s}^{*}=\min \sum_{n \in V_{D}} c_{n}^{\mathrm{e} \& \mathrm{~s}} \sum_{t=1}^{T_{s}} \Delta p_{n t s}
$$

subject to

$$
\sum_{i \in V \cup L^{0}} c_{i}^{\mathrm{har}} w_{i}+\sum_{l \in L^{\prime}} c_{l}^{\mathrm{inv}} v_{l} \leq B
$$

(8)-(22) for the given scenario $s$

$$
\begin{aligned}
& v_{l}, w_{i} \in\{0,1\} \quad \forall l \in L^{\prime} \forall i \in L^{0} \cup V \\
& r_{i t}, x_{i t} \in\{0,1\} \quad \forall i \in L^{0} \cup V \forall t=1 \ldots T_{s} .
\end{aligned}
$$

The objective function in $p$-CIEPP states that DMs are more interested in obtaining a solution that performs well under nominal operation conditions, while the $p$-robustness constraints (6) suggest that they are willing to make additional investments in CI hardening and expansion to protect against potential disruptions to the CI facilities.

The proposed $p$-CIEPP framework is applicable to singlecommodity CIs, e.g., power, water, wastewater, and supply chain systems. However, the current formulation of the $p$-CIEPP problem assumes that the link flows can be arbitrarily controlled, which is not the case for power systems [28]. Therefore, additional constraints representing the second Kirchhoff's law must be embedded into the framework to account for the physics of the service provided through the power network. The dc power flow model is a commonly used linear approximation of the power infrastructure operations. It has been proven to be able to give good results for active power flows in high-voltage transmission systems [47] and is widely used in system expansion and protection planning [18], [41], [48]. Therefore, in our case study for power infrastructures, we incorporate a set of dc power flow constraints into the $p$-CIEPP problem in the same way as in [7], [28], and [49].

\section{Problem Complexity}

The proposed $p$-CIEPP model is a two-stage mixed-integer linear program (MILP) problem, which is NP-hard [50], as stated in Proposition 1. Actually, multilevel models are generally very difficult to solve [51]. Hansen et al. [52] proved that even the simplest bilevel models, the ones with continuous variables on every level, are strongly NP-hard. The $p$-CIEPP has a block diagonal structure that includes coupling variables between the blocks. The first-stage (coupling) variables are the system planning variables $\boldsymbol{v}$ and $\boldsymbol{w}$, and the second-stage variables include postdisruption system restoration scheduling and dispatching variables $\boldsymbol{r}, \boldsymbol{x}, \boldsymbol{f}, \boldsymbol{g}, \Delta \boldsymbol{p}$.

Proposition 1: The p-CIEPP model is NP-hard.

This should not be surprising since NP-hardness is a typical feature of two-stage programming problems, where different uncertainty scenarios are considered in the second stage [53]. However, what makes the problem even more challenging to solve is that the feasibility problem, i.e., determining whether a given instance of the $p$-CIEPP is feasible, is itself NP-complete.

Proposition 2: For $|S| \geq 1$ and $p \geq 0$, the feasibility problem for the $p$-CIEPP is NP-complete.

Proof: First, the feasibility problem is in NP since the feasibility of a given solution can be checked in polynomial time. To prove NP-completeness, we reduce the $p$-CIEPP to the $p$-robust 


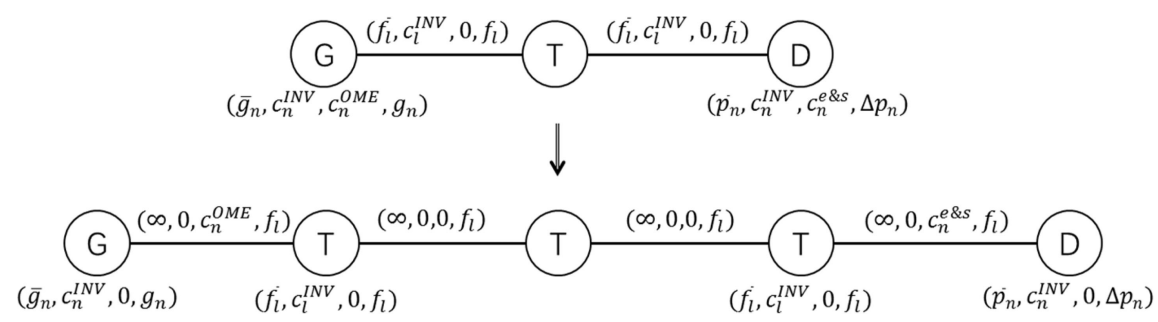

Fig. 2. Example of an auxiliary graph.

logistics network design problem ( $p$-LNDP) proposed by Peng et al. [45], whose feasibility problem is NP-complete. Note that $C_{\mathrm{OPC}}$ can be rewritten as

$$
\begin{aligned}
C_{\mathrm{OPC}}= & \sum_{n \in V_{G}}\left(c_{n}^{\mathrm{o} \& \mathrm{~m}}+c_{n}^{\mathrm{emi}}\right) \\
& \left(\sum_{l \in L \mid o(l)=n} f_{l}-\sum_{l \in L \mid d(l)=n} f_{l}\right)+\sum_{l \in L} c_{l}^{\mathrm{o} \& \mathrm{~m}} f_{l} \\
& +\sum_{n \in V_{D}} c_{n}^{\mathrm{e \& s}}\left(\bar{p}_{n}-\sum_{l \in L \mid d(l)=n} f_{l}+\sum_{l \in L \mid o(l)=n} f_{l}\right) .
\end{aligned}
$$

Then, we construct an auxiliary graph as follows. For any one of the transmission lines, we replace it with a dummy node and two new transmission lines. The dummy node has exactly the same characteristics as the original transmission line. The two new lines have infinite capacity, zero investment cost (economic and societal cost of investment in hardening or expansion is denoted here as $c^{\mathrm{INV}}$ and called investment cost for simplicity), and zero unit flow cost. However, if the new line is connected to a supply node, its unit flow cost is set as that of the supply node (the unit flow cost of a supply node $n \in V_{G}$ is defined as the sum of its $\mathrm{O} \& \mathrm{M}$ cost and emission cost, i.e., $c_{n}^{\mathrm{OME}}=c_{n}^{\mathrm{o} \& \mathrm{~m}}+c_{n}^{\mathrm{emi}}$ ); if the new line is connected with a demand node $n \in V_{D}$, its unit flow cost is set as $c_{n}^{\text {e\&s }}$ of the demand node. Fig. 2 gives an example consisting of the original graph and its auxiliary graph; the symbols in the parentheses are capacity (expected demand for demand nodes), investment cost, unit flow cost, and actual flow, respectively.

Then, the original problem is equivalent to minimizing the sum of investment and network flow costs, and if $T_{s}=1$ (i.e., there is no restoration process), the $p$-CIEPP reduces to the $p$ LNDP proposed by Peng et al. [45], whose feasibility problem is proven to be NP-complete. Since $T_{s}=1$ is a special case of the $p$-CIEPP, the feasibility problem of the general case of the $p$-CIEPP is at least NP-complete. We have already shown that the feasibility problem of the $p$-CIEPP is in NP; therefore, Proposition 2 holds.

Consequently, unlike most other two-stage robust or stochastic optimization problems, it can be challenging just to find a feasible solution for the proposed $p$-CIEPP.

\section{Defining Scenario Set of SLDs}

Natural disasters (e.g., earthquakes, hurricanes, and other extreme weather) and malicious attacks are generally highly uncertain events that are difficult to estimate, model, and predict.
In this paper, we consider potential disaster events represented by SLDs [17], [54], i.e., disruptions that cause direct damage or interruption of system components, which exist within some localized area, while those outside this area remain operating. For example, on August 12, 2015, the Tianjin chemical explosion event in China caused the damage or interruption of some main road segments, a subway station, and many other infrastructure components located within $1 \mathrm{~km}$ from the explosion center. On January 3, 2018, the strong winds generated by Eleanor storm caused all the train services to be interrupted throughout Normandy (the affected area can be seen as a concentric zone centered on Lisieux and with a radius of around $100 \mathrm{~km}$ ), but the railway interruption was still localized from the whole country view. The 9/11 New York terrorist attacks caused the full collapse of the WTC1 and the WTC2, and massive debris caused the damage and interruption of many infrastructure components within $0.4 \mathrm{~km}$ from the attack center, including power substations, water pipes, telecommunication centers, and subway tunnels. It can be understood from the above examples that the SLDs can be triggered by different origins, e.g., natural hazards, human accidents, or intentional attacks. It should be noted that other types of disruption scenarios could also be considered in the proposed $p$-CIEPP model, depending on the particular CI systems of interest.

The uncertainty of SLDs is modeled through a set of scenario $S$, where each scenario represents an attack plan from natural disaster occurrences or terrorist attacks. The scenario set $S$ is made up of vectors $a_{s}$ of $0 \mathrm{~s}$ and $1 \mathrm{~s}$ as follows:

$$
a_{s}=\left\{a_{1 s}, \ldots, a_{M_{1} s}, a_{\left(M_{1}+1\right) s}, \ldots, a_{\left(M_{1}+M_{2}\right) s}\right\}
$$

where $M_{1}=|V|, M_{2}=\left|L^{0}\right|$, and $a_{i s}$ is a constant equal to 0 if facility $i$ is attacked in scenario $s$, being 1 otherwise. The geographical vicinity of the disruption is characterized by assuming that all facilities being attacked are within a distance $R_{h}$ from a random location in the system [55]

$$
\operatorname{Dist}(i, \operatorname{centr}(s)) \leq R_{h} \quad \forall a_{i s}=0 \forall s \in S
$$

where centr $(s)$ represents the epicenter location of the disruption $s$ and $\operatorname{Dist}(i, j)$ is the geographical distance between $i$ and $j$. Fig. 1(b) presents an illustrative SLD scenario, whose epicenter is located at D5 and with an influence radius of $R_{h}$. An unprotected line will be considered as failed even if only partially within the disruption area, e.g., the line G2-D6 in Fig. 1(b).

The scenario set $S$ can be generated in various ways for different systems. For simplicity, this paper considers the case with $M$ scenarios, and in each scenario, one node $i \in V$ in the system is randomly chosen as the epicenter of disruption, and all the facilities within the circle with center at $i$ and radius equal to $R_{h}$ 
are regarded as damaged. Since every CI system is unique, every utility will have a specific set of "what-if" contingencies that it is interested in considering. DMs can decide which scenarios are most likely to occur or are of importance to plan against, in practice. For example, models for specific natural disasters, such as earthquakes [56] and storms [57], can be used to identify the disruption scenarios by examining what the potential threats to the CIs are and how the system components are affected; this includes the so-called game-theoretical interdiction models for intentional attacks [12], [58]. These models are outside the scope of this paper and will be considered in our future research.

\section{AlgORITHM DEVELOPMENT}

In this section, we first develop an exact algorithm framework based on scenario decomposition for the solution of the $p$-CIEPP. Then, a metaheuristic search algorithm is proposed for efficiently solving the proposed $p$-CIEPP model by combining the exact SBD with the heuristics of VNS.

\section{A. Scenario-Based Decomposition}

Decomposition is usually used for solving complex MILP problems with a block diagonal structure that includes coupling variables between the blocks. Algorithms based on Lagrangian relaxation and Benders decomposition have been proposed to solve $p$-robust network design problems [44], [46]. However, these approaches can be computationally expensive, especially when the number of scenarios is large [45]. Here, an SBD method can be applied to the proposed $p$-CIEPP model (5)-(26) due to the following important observation.

Remark 1: All the scenario-dependent decision variables do not appear in the objective function of the proposed p-CIEPP model; therefore, any optimal planning solution $\boldsymbol{v}$ and $\boldsymbol{w}$ based on a subset of disruption scenarios $S^{\prime} \subset S$ and feasible for all considered scenarios $S$ is an optimal solution of the p-CIEPP model.

Based on this observation, we can develop an SBD scheme to solve the $p$-CIEPP model. The basic idea of this algorithm is to solve problems with iteratively larger sets of scenarios until a solution is obtained that is feasible for all remaining scenarios. A similar idea can be found in [59]. The detailed steps are described as follows.

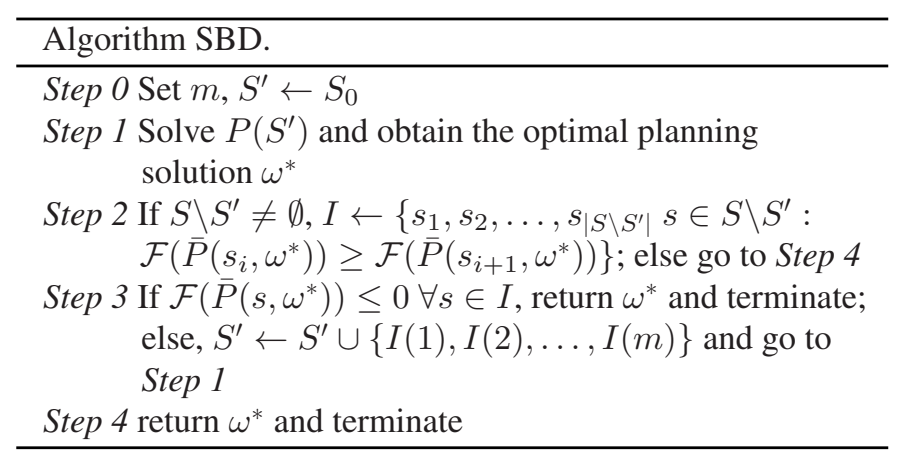

The algorithm initially sets parameter $m$ and takes as input the set of disruption scenarios and an initial subset of scenarios to consider, $S^{\prime}$. Step 1 solves $p$-CIEPP on $S^{\prime}$, where $P\left(S^{\prime}\right)$ and $\omega^{*}$ are used to denote the $p$-CIEPP problem based on $S^{\prime}$ and the optimal planning solution, respectively. Step 2 then evaluates $\omega^{*}$ on the remaining scenarios in $S \backslash S^{\prime}$ if it is not empty. Set $I$ contains all the remaining scenarios and is descending sorted by the function value $\mathcal{F}\left(\bar{P}\left(s, \omega^{*}\right)\right)$ of each scenario $s$. The function $\mathcal{F}: \bar{P}\left(s, \omega^{*}\right) \rightarrow \mathbb{R}_{+}$measures the degree of infeasibility of the problem $\bar{P}\left(s, \omega^{*}\right)$ that is 0 if the problem $\bar{P}\left(s, \omega^{*}\right)$ is feasible, i.e., $\omega^{*}$ is feasible in terms of constraints (6)-(26), positive otherwise. Particularly, this is implemented by minimizing the system resilience loss, i.e., the total cumulative unsatisfied demand across, for each of the given disruption scenario $s$. A positive function value $\mathcal{F}\left(\bar{P}\left(s, \omega^{*}\right)\right)$ is determined by the minimum system resilience loss when the planning decision $\omega^{*}$ and the disruption scenario $s$ are given. This function prices the current solution over all remaining scenarios $s \in S \backslash S^{\prime}$. If all prices are 0 , then the algorithm terminates with solution $\omega^{*}$. Otherwise, the algorithm adds the first $m$ scenarios in set $I$ with the top $m$ worst infeasibility measures to $S^{\prime}$ (Step 3).

\section{B. Variable Neighborhood Search}

The most computationally demanding step in the SBD algorithm is to solve the problem $P\left(S^{\prime}\right)$ in Step 1. It can also be time-consuming when the number of considered scenarios becomes larger. To overcome this limitation, we develop a search heuristic method based on VNS [36], [60]-[62]. VNS is a powerful metaheuristic, which is based on the systematic change of neighborhoods toward the local optima and out of the regions that contain them. The algorithm first fixes a subset of first-stage decision variables $\boldsymbol{v}$ and $\boldsymbol{w}$ to their current values and then searches the residual variables in order to obtain a better solution. In fact, if all the decision variables in the first stage are fixed, the problem is simply decomposed into $|S|$ separate subproblems that can be solved with much less computational efforts. More formally, we let $P(\omega, \mathcal{Z})$ denotes the problem with a subset of first-stage variables, let $\mathcal{Z} \subset\{\boldsymbol{v}, \boldsymbol{w}\}$ fixed to $\omega$, i.e., $z_{i}=\omega\left(z_{i}\right)$, and $P^{\mathrm{LP}}$ represents the LP relaxation of problem $P$. Then, the detailed procedure of VNS is given as follows.

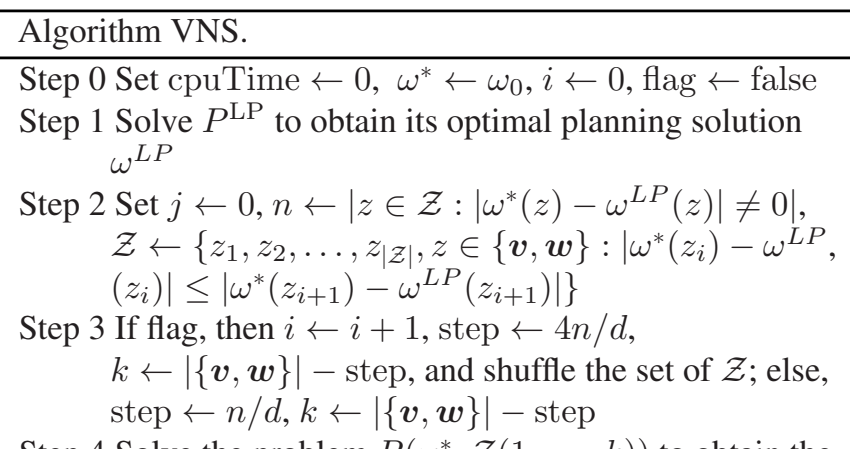

Step 4 Solve the problem $P\left(\omega^{*}, \mathcal{Z}(1, \ldots, k)\right)$ to obtain the solution $\omega^{\prime}$

Step 5 If $f\left(\omega^{\prime}\right)<f\left(\omega^{*}\right)$, i.e., $\omega^{\prime}$ obtains a smaller objective value than $\omega^{*}$, then $\omega^{*} \leftarrow \omega^{\prime}, i \leftarrow 0$, flag $\leftarrow$ false, $j \leftarrow$ maxIter; else, $j \leftarrow j+1, k \leftarrow k-$ step $/ 2$, and if $j>$ maxIter, then flag $\leftarrow$ true.

Step 6 If cpuTime $<$ maxTime, $k \geq 0$ and $j<$ maxIter, go to Step 4; else, go to Step 7

Step 7 If cpuTime $<$ maxTime and $i<\operatorname{maxStart,\text {go}}$ to Step 2; else, terminate the algorithm and return $\omega^{*}$ 
The algorithm starts with a feasible solution $\omega_{0}$ and Step 1 solves the LP relaxation of the $p$-CIEPP to obtain $\omega^{\mathrm{LP}}$. Then, in Step 2, the number of variables assignments that are variant between the solution $\omega^{\mathrm{LP}}$ and the best-known solution $\omega^{*}$ is counted, and the elements in set $\mathcal{Z}$ are ordered by the difference between their assignments in $\omega^{*}$ and $\omega^{\mathrm{LP}}$, where $\omega(z)$ denotes the variable assignment of $z$ in solution $\omega$. Based on whether the algorithm is in a restart mode indicated by the variable flag in Step 3, the algorithm will update the rate at which the size of the neighborhood is increased. If the algorithm is in a restart, the order of the variables in $\mathcal{Z}$ is shuffled. Step 4 computes the best solution in the neighborhood of $\omega^{*}$, where the first $k$ elements of $\mathcal{Z}$ are fixed. If the resulting solution from Step 4 is better, i.e., the objective value from $\omega^{\prime}$ is smaller than that from $\omega^{*}\left(f\left(\omega^{\prime}\right)<f\left(\omega^{*}\right)\right)$, the algorithm updates the currently best-known solution $\omega^{*} \leftarrow \omega^{\prime}$ and proceeds. Otherwise, the neighborhood size of $\omega^{*}$ is increased by $k \leftarrow k-$ step $/ 2$. This neighborhood search iterates until the predefined maximum number of neighborhood resizing, maxIter, is reached, or a CPU time limit, maxTime, is reached (Step 6). The algorithm terminates when a predefined maximum number of restart times, maxStarts, is reached, or a CPU time limit, maxTime, is reached (Step 7). This paper sets $\operatorname{maxStart}=5$, maxTime $=10 \mathrm{CPU}$ hours, maxIter $=4$, and $d=2$.

\section{Hybrid SBD and VNS Algorithm}

Given that the problem $P\left(S^{\prime}\right)$ in the SBD algorithm Step 1 can be solved by the VNS heuristics, the two methods can be hybridized to solve the $p$-CIEPP model. The hybrid algorithm proceeds the same as the SBD framework, except that the VNS algorithm is applied in Step 1 of the SBD algorithm for the solution of $P\left(S^{\prime}\right)$.

\section{Application to A Power Transmission System}

\section{A. Dataset}

A test network model based on the British high-voltage power transmission system is considered to study the proposed $p$ CIEPP model. The original power system includes the 400- and $275-\mathrm{kV}$ transmission levels, and the reduced representative network comprises 29 nodes and 50 lines, as shown in Fig. 3. Technical data for this system can be found in [63]. Table I provides data for the productions and demands. Each production unit is characterized by its maximum generation capacity $\bar{g}_{n}$ (Column 2), the O\&M cost for unit power generation $c_{n}^{\mathrm{o} \& \mathrm{~m}}$ (Column 3), and unit emission $\operatorname{cost} c_{n}^{\mathrm{emi}}$ (Column 4). Similarly, each demand node is characterized by its expected value $\bar{p}_{n}$ (Column 6) and its economic and societal cost of unit load shedding $c_{n}^{\text {e\&s }}(\mathrm{Col}-$ umn 7). Note that the case study assumes that the O\&M cost associated with a unit of power flow through transmission line $l \in L$ is 0 .

Data regarding candidate lines to be potentially built are provided in Table II, where the last column gives the annualized investment costs for each of these lines. The annualized investment costs are assumed with reference to preevious studies [49],

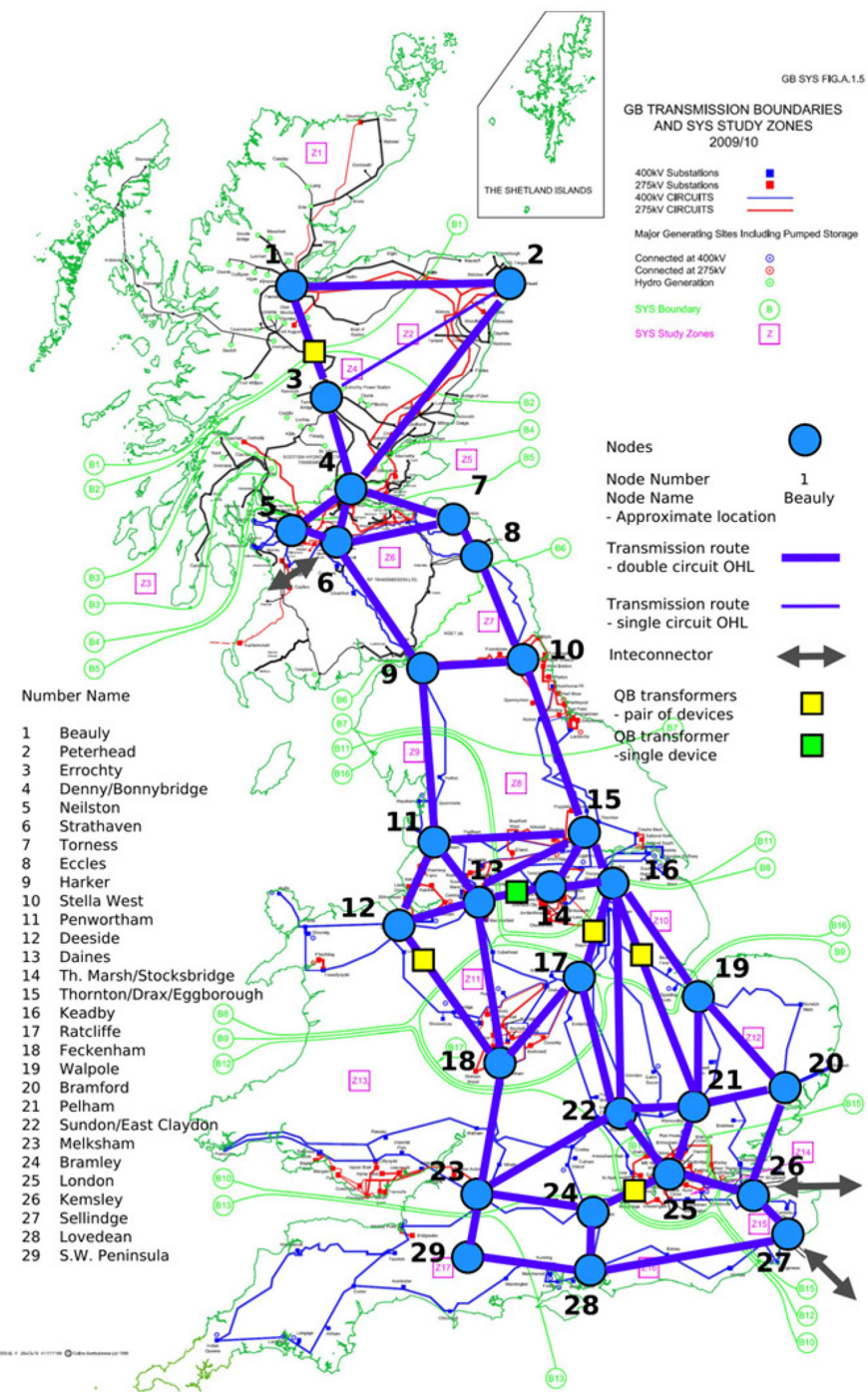

Fig. 3. Test network model of the British electric power transmission system [66].

[64], which generally scale linearly with physical line length. Besides, we fix parameter $\sigma$ in (5) to $\sigma=8760$, which is the total number of hours in one year. This allows considering the same scale for both operation costs (hourly basis) and annualized investment costs. The hardening/defense cost for a power transmission line depends on the types of defense measures (e.g., using underground cables instead of overhead lines, upgrading poles and structures with stronger materials, tree trimming and vegetation management, building the substation sites with new structures like walls and floodgates, etc.) and can vary from system to system. In this paper, it is assumed to be a constant value for each existing transmission line, $c_{l}^{\text {har }}=\$ 0.5$ million, for simplicity, while considering the order of magnitude of the structural hardening cost in practice [65]. Nevertheless, the defense cost functions will have to be validated and calibrated when applying the proposed model to specific systems. This is possible as it is the system operator that makes the decisions about the allocation of the defense resources. 
TABLE I

DATA FOR GENERATION AND DEMAND NODES

\begin{tabular}{lllllll}
\hline \hline Gen. ID & $\bar{g}_{n}(\mathrm{GW})$ & $c_{n}^{\text {o\&m }}\left(10^{3} \$ / \mathrm{GWh}\right)$ & $c_{n}^{\text {emi }}\left(10^{3} \$ / \mathrm{GWh}\right)$ & $\mathrm{Dem} . \mathrm{ID}$ & $\bar{p}_{n}(\mathrm{GW})$ & $c_{n}^{\text {ess }}\left(10^{3} \$ / \mathrm{GWh}\right)$ \\
\hline 1 & 0.4 & 0.45 & 0.45 & 6 & 0.5 & 50 \\
2 & 0.9 & 0.55 & 0.55 & 8 & 0.2 & 50 \\
3 & 0.2 & 0.60 & 0.60 & 9 & 0.2 & 50 \\
4 & 1.2 & 0.50 & 0.50 & 13 & 3.3 & 50 \\
5 & 0.4 & 0.50 & 0.50 & 14 & 2.4 & 50 \\
7 & 1.5 & 0.50 & 0.50 & 18 & 5.0 & 50 \\
10 & 0.16 & 0.50 & 0.50 & 21 & 0.25 & 50 \\
11 & 1.3 & 0.55 & 0.55 & 22 & 2.0 & 50 \\
12 & 2.6 & 0.40 & 0.40 & 24 & 2.0 & 50 \\
15 & 4.3 & 0.50 & 0.50 & 25 & 10.5 & 50 \\
16 & 10 & 0.55 & 0.55 & 28 & 2.1 & 50 \\
17 & 0.5 & 0.60 & 0.60 & 29 & 1.2 & 50 \\
19 & 0.6 & 0.60 & 0.60 & & & \\
20 & 0.2 & 0.50 & 0.50 & & & \\
23 & 1.5 & 0.50 & 0.50 & & & \\
26 & 3.6 & 0.50 & 0.50 & & & \\
27 & 0.7 & 0.50 & 0.50 & & & \\
\hline \hline
\end{tabular}

TABLE II

DATA FOR CANDIDATE LINES

\begin{tabular}{ccc}
\hline \hline Candidate line & $\bar{f}_{l}(\mathrm{GW})$ & Annual investment cost $c_{l}^{\text {inv }}$ (million \$) \\
\hline $7-9$ & 1.0 & 0.8 \\
$9-15$ & 1.0 & 0.9 \\
$10-11$ & 1.0 & 0.9 \\
$17-19$ & 1.0 & 0.8 \\
$18-23$ & 1.0 & 0.6 \\
$22-24$ & 1.0 & 0.5 \\
$23-28$ & 1.0 & 0.7 \\
$25-28$ & 1.0 & 0.6 \\
\hline \hline
\end{tabular}

This paper considers different numbers, $M$, of SLD scenarios with an influencing radius $R_{h}=100 \mathrm{~km}$ to represent potential natural hazards in the studied areas (e.g., storms in Great Britain). The different sets of disruption scenarios are generated randomly and in an incremental way, i.e., scenarios in small sets (e.g., $M=5$ ) are also included in large sets (e.g., $M=10$ ). Only transmission lines are failed during disruptions. The repair crew is the limited resource that is allocated to repair damaged lines. The expected repair time is based on historical data of power system restoration [67], [68].

The algorithms are implemented using the CPLEX MATLAB API and executed on a computer equipped with 16-GB RAM, 500-GB memory, and a quad-core 3.6-GHz Intel I7-4790 processor. The MIP solver used is CPLEX 12.6.1 with default parameter settings.

\section{B. Computational Results and Analysis}

First, we investigate the "price of resilience," in other words, how much it costs to design a more resilient system that has much lower resilience loss when disruptive events occur. Fig. 4 shows the tradeoff curves between the nominal cost (the objective value (3) that includes the annualized investment cost and total system

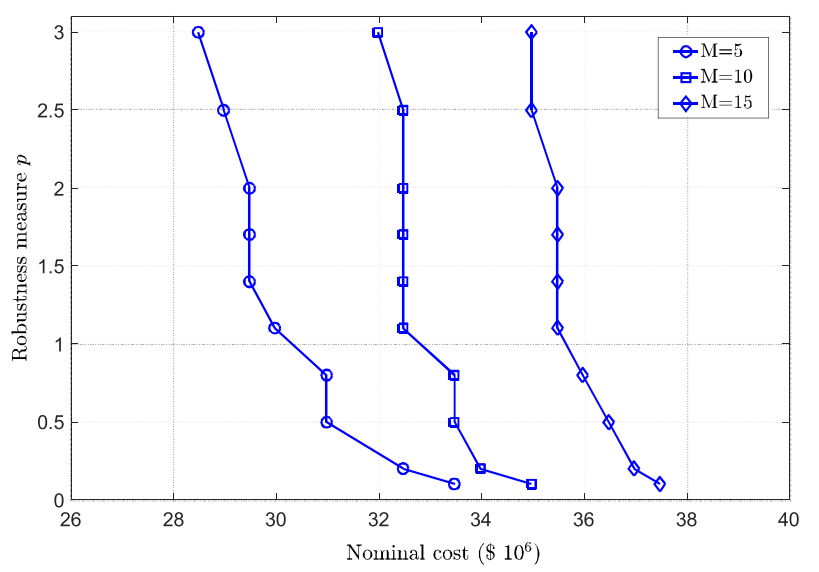

Fig. 4. Tradeoff between the robustness measure $p$ and the nominal cost. The parameter $M$ indicates the number of disruption scenarios. A higher value of $p$ along the vertical axis means a larger regret in system resilience loss, thus a less resilient system.

operating costs under nominal condition) and the value of $p$ for different numbers of disruption scenarios, $M=5,10$, and 15. The influencing radius $R_{h}=100 \mathrm{~km}$, and the number of repair crew is one $K=1$ for all the three cases. Note that $p$ measures the maximal relative regret about system resilience loss, and higher value of $p$ means larger regret in system resilience loss, thus a less resilient system. It can be found that the left-most portions of the curves are rather steep, which means that the maximal relative regret can be largely reduced with only a small increase in the nominal cost. For instance, in $M=15$, we are able to reduce the maximum relative regret from 3 by $63.3 \%$ to 1.1 , with a slight increase in cost from \$34.4734 to \$35.4734 million, or $2.9 \%$. In other words, with little additional investment, one can obtain CI systems that are more resilient to SLDs. Besides, 
TABLE III

INVESTMENT RESULTS FOR $M=5$ AND DIFFERENT $p$

\begin{tabular}{lllll}
\hline \hline & \multicolumn{4}{c}{$p$} \\
\cline { 2 - 5 } & 2.5 & 1.5 & 1.0 & 0.5 \\
\hline$C_{O P C}(\mathrm{M} \$)$ & 28.97 & 29.47 & 29.97 & 30.97 \\
$C_{I N V}(\mathrm{M} \$)$ & 3.0 & 3.5 & 4.0 & 5.0 \\
Max. scenario costs (M\$) & 14.04 & 9.12 & 5.32 & 3.01 \\
& & $9-11,11-13$, & $11-13,11-12$, & $9-11,11-13,12-13$, \\
& $11-13,11-15$, & $12-18,14-16$, & $11-15,12-18$, & $13-18,14-15,16-19$, \\
\multirow{2}{*}{ Set of lines to be hardened } & $12-18,14-15$, & $21-25,16-22$, & $14-16,21-25$, & $21-25,16-22,23-24$, \\
& $23-24,25-26$ & $25-26$ & $16-22,25-26$ & $25-26$ \\
\hline \hline
\end{tabular}

one can find from Fig. 4 that for each fixed $p$, considering a larger number of disruption scenarios results in an increased nominal cost, which is due to the fact that more investments in system hardening and expansion have to be carried out in order to protect the system from additional disruptions. That is, investment decisions are fairly sensitive to the considered scenario set of SLDs. This suggests that DMs should decide carefully which disruption scenarios are most likely to occur in a specific system.

Table III reports the investment results obtained for the scenario set $M=5$ under different values of $p$. Row 3 gives the nominal costs, i.e., the objective values, of the obtained systems, and Row 4 provides the investment costs. Row 5 represents the costs of system unmet demand under the worst disruption scenario $\left(\operatorname{argmax}_{s} \sum_{n \in V_{D}} c_{n}^{\text {e\&s }} \sum_{t=1}^{T_{s}} \Delta p_{n t s}\right)$, and the last row identifies the lines to be protected for each of the cases analyzed. The investment costs increase with the decreasing $p$ as additional lines are chosen to be hardened, resulting in increased nominal costs. However, this relative little additional investment gains a large reduction in the maximal scenario cost, i.e., the penalty cost for unmet system demand under the worst disruption scenario, which represents the maximal system resilience loss under all the scenarios. In other words, with little additional investment, one can obtain a large improvement in the system resilience against the worst disruption scenario. Furthermore, the nonincremental nature of the investment plan can be observed by increasing the robustness level (decrease of $p$ ). That is to say, the optimum set of lines to be protected in the small investment case is not necessarily a subset of the lines to be protected in the large investment cases. For example, the lines 11-15, 14-15, and 23-24 are protected for the optimum planning if $p=2.5$, but they are replaced by lines 9-11, 14-16, 21-25, and 16-22 if $p=1.5$.

The above results are all obtained for only one repair crew $K$ $=1$; we then analyze the impact of different numbers of repair crews on the optimization results. Fig. 5 shows how the nominal costs and maximal scenario costs change under different number of repair crews $K$ for $M=5$ and $p=0.5$. From this figure, it can be found that increasing the number of repair crews can lead to a reduction in the maximal scenario costs, i.e., the penalty cost

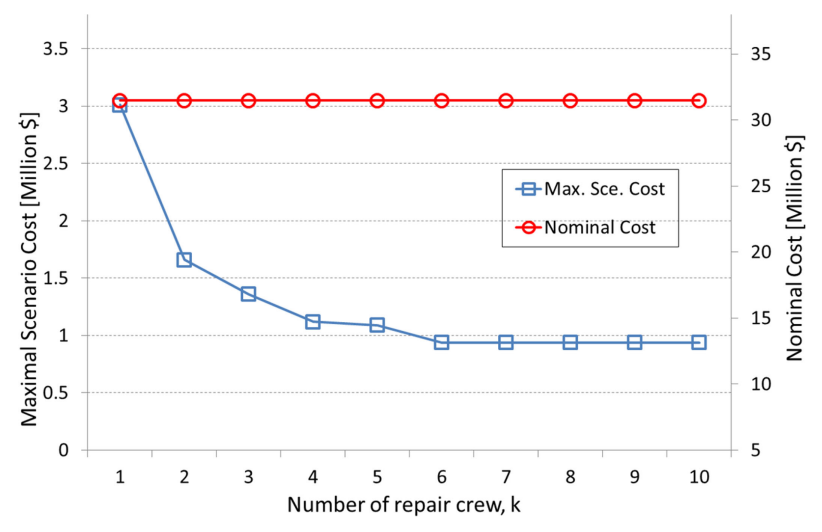

Fig. 5. Nominal costs and maximal scenario costs evolve along with different repair crews $K$ for $M=5$ and $p=0.5$.

for unmet system demand under the worst disruption scenario, while the nominal cost keeps unchanged. For example, the maximal scenario cost is reduced from $\$ 3.01$ to $\$ 1.66$ million when $K$ increases from 1 to 2 , and it is reduced to $\$ 1.36$ million when $K=3$. However, the mitigation effectiveness of maximal scenario cost becomes less significant when $K$ becomes larger, and the maximal scenario cost keeps unchanged when $K>6$. The results show that increasing the number of repair crews $K$, within a certain region, is also an effective defense strategy to enhance system resilience against disruptions. As long as the DMs are aware of the (marginal) cost of deploying one unit of repair crew, they can decide the optimum number of repair crews, e.g., by comparing the deployment cost and the reduction of the maximal scenario cost. The nominal cost (objective value) remains constant, and the optimization obtains the same investment decision under different $K$. This is probably because increasing $K$ may mitigate the penalty cost for each scenario, i.e., the lefthand side of constraints (6), but it also leads to a reduction of the optimal penalty $\operatorname{costs} C_{s}^{*}$ in the right-hand side of constraints (6). Therefore, the relative regret under each scenario may keep unchanged. This result shows that the investment decisions of the $p$-CIEPP model are not sensitive to the number of repair crews, given that it is determined before the planning. 
TABLE IV

COMPutational Performance OF THE Proposed Algorithm

\begin{tabular}{|c|c|c|c|c|c|c|}
\hline \multirow{2}{*}{$M$} & \multirow{2}{*}{$p$} & \multicolumn{2}{|c|}{ CPLEX } & \multicolumn{2}{|c|}{ SBD-VNS } & \multirow{2}{*}{$\begin{array}{c}\text { Comparison } \\
\text { Time Saving }\end{array}$} \\
\hline & & Time (s) & Obj./Gap & Time (s) & Obj. & \\
\hline \multirow{7}{*}{5} & 0.2 & 1844 & 32.47 & 179 & 32.47 & $90 \%$ \\
\hline & 0.5 & 991 & 30.97 & 101 & 30.97 & $90 \%$ \\
\hline & 0.8 & 1233 & 30.97 & 139 & 30.97 & $89 \%$ \\
\hline & 1.1 & 646 & 29.97 & 155 & 29.97 & $76 \%$ \\
\hline & 1.4 & 1114 & 29.47 & 147 & 29.47 & $87 \%$ \\
\hline & 1.7 & 1424 & 29.47 & 106 & 29.47 & $93 \%$ \\
\hline & 2.0 & 1317 & 29.47 & 289 & 29.47 & $78 \%$ \\
\hline \multirow{7}{*}{10} & 0.2 & 1285 & 33.97 & 352 & 33.97 & $73 \%$ \\
\hline & 0.5 & 1532 & 33.47 & 313 & 33.47 & $80 \%$ \\
\hline & 0.8 & 2514 & 33.47 & 579 & 33.47 & $77 \%$ \\
\hline & 1.1 & 1475 & 32.47 & 297 & 32.47 & $80 \%$ \\
\hline & 1.4 & 1076 & 32.47 & 215 & 32.47 & $80 \%$ \\
\hline & 1.7 & 814 & 32.47 & 371 & 32.47 & $54 \%$ \\
\hline & 2.0 & 948 & 32.47 & 246 & 32.47 & $74 \%$ \\
\hline \multirow{7}{*}{15} & 0.2 & 3600 & $36.97 / 1.40 \%$ & 493 & 36.97 & $80 \%$ \\
\hline & 0.5 & 3600 & $36.47 / 2.96 \%$ & 652 & 36.47 & $86 \%$ \\
\hline & 0.8 & 3600 & $35.97 / 1.70 \%$ & 581 & 35.97 & $82 \%$ \\
\hline & 1.1 & 3600 & $35.47 / 4.36 \%$ & 850 & 35.47 & $84 \%$ \\
\hline & 1.4 & 3600 & $35.47 / 1.56 \%$ & 667 & 35.47 & $76 \%$ \\
\hline & 1.7 & 3600 & $35.47 / 2.33 \%$ & 653 & 35.47 & $81 \%$ \\
\hline & 2.0 & 3600 & $35.47 / 3.55 \%$ & 774 & 35.47 & $82 \%$ \\
\hline
\end{tabular}

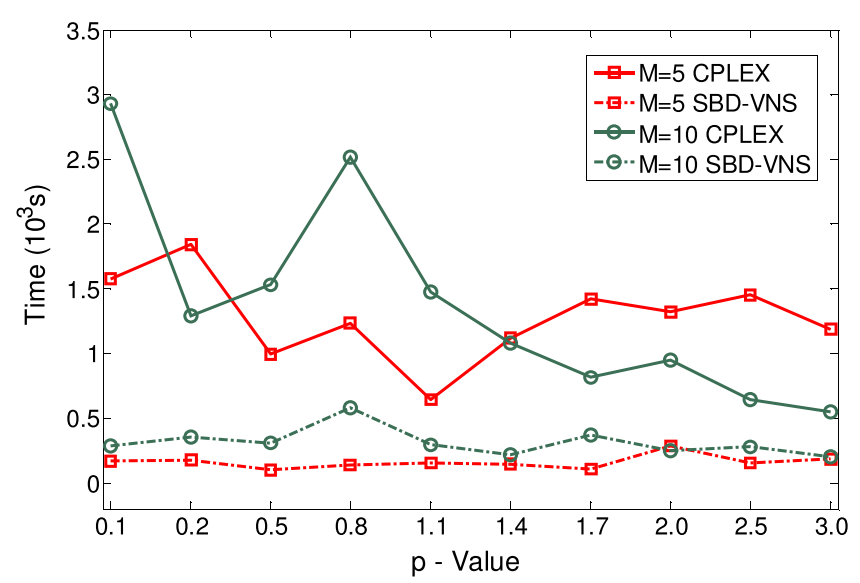

Fig. 6. Algorithm performance with different values of $p$.

Finally, we compare the computational performance of our algorithm with that of CPLEX in Table IV. Each row with a different combination of $M$ and $p$ gives an experiment instance. For each algorithm, two columns report the runtime ("Time") and objective value ("Obj."). The optimality gap with against the CPLEX lower bound ("Gap") is also reported when CPLEX does not converge after $1 \mathrm{~h}$ running. The proposed SBD-VNS algorithm is compared with CPLEX, and the last "Time" column gives the percentages of CPLEX's CPU time required by our algorithm. From the table, one can find that our algorithm takes only a small fraction of CPLEX's time (20\% on average) to find the same solution. All the instances of $M=15$ could not converge in $3600 \mathrm{~s}$ for CPLEX. Furthermore, Fig. 6 visualizes how the algorithm performance evolves with different $p$ for the dataset $M=5$ and $M=10$, for which CPLEX solves to convergence.
It shows that the proposed SBD-VNS algorithm is not affected much by the value of $p$, while CPLEX's solution times are much affected by different $p$.

To complement this paper and investigate the applicability of the proposed method, a large system (the Swiss 380/220-kV high-voltage power transmission system) comprising 161 nodes and 210 lines [10], [69] has been considered, and more disruptive scenarios on it have been analyzed.

Table $\mathrm{V}$ provides the results obtained on this additional network. Different numbers of disruption scenarios, $M=5,10$, $15,20,25$, and 30 with an influencing radius $R_{h}=20 \mathrm{~km}$ are considered. The number of repair crews is set as $K=4$. Parameter $p$ is set as 3.0, rendering all the considered instances feasible. In Table V, columns 2-4 report the size of the formulated problem represented by the number of variables, the number of binary variables, and the number of constraints. It shows that the problem size generally increases linearly with the number of the scenarios considered. For all scenarios, solving the MIP problem directly by CPLEX could not converge in $3600 \mathrm{~s}$ and with gaps larger than $10 \%$ due to the very large problem size. Columns 5 and 6 report the objective values obtained and computational times needed by the proposed SBD-VNS algorithm. It can be seen that the computational time increases largely compared with the corresponding cases in the previous test network in Table IV. Actually, it is a common feature of branch-and-cutbased algorithms when addressing MIP problems. Nonetheless, for large-scale networks, the proposed planning problem can be solved offline, and the issue of CPU time can be tackled by allowing adequate computational time. Also, the computational complexity can be possibly decreased through approaches like the distributed computing technique, which could be interesting future research.

\section{Managerial Insights and Discussions}

The proposed $p$-robust optimization model serves as a supporting tool for making decisions on CI system planning, and both resilience and nominal cost are considered to measure the consequences of the decisions. The nominal cost focuses on relatively certain consequences that are distributed over the life cycle and can be predicted with good approximation. Resilience considers large consequences associated with disruptive events with small probabilities of occurrence, which are typically difficult to estimate. In practice, DMs are usually more concerned with minimizing the nominal operation cost and wish to limit the regret in each possible contingency scenario so as to bound system exposure to risk. In this light, the framework of the proposed $p$-robust optimization appears appropriate to model the decision problems for CI systems and can lead to a solid and systematic integration of resilience into system planning.

The previous section shows that, for the tested system, a large improvement in resilience against SLDs can be achieved with very little additional monetary investment in transmission line hardening. Although this result is not necessarily true for other CI systems, a tradeoff between nominal cost and resilience does exist. In such scenarios, a comprehensive analysis of all the possible outcomes of each decision is of utmost importance for 
TABLE V

Computational Results on the Swiss High-Voltage 380/220-KV Power Transmission Network FOR DiFFERENT SCENARIOS FOR $p=3.0$

\begin{tabular}{cccccc}
\hline \hline \multirow{2}{*}{$\begin{array}{c}\text { No. of } \\
\text { scenarios } M\end{array}$} & \multicolumn{3}{c}{ Problem Size } & \multicolumn{2}{c}{ SBD-VNS } \\
\cline { 2 - 6 } & No. of var. $\left(10^{3}\right)$ & No. of bin var. $\left(10^{3}\right)$ & No. of cstr. $\left(10^{3}\right)$ & Obj. & Time (s) \\
\hline 5 & 275.9 & 115.7 & 277.8 & 6342.7 & 327.5 \\
\hline 10 & 555.1 & 231.2 & 555.5 & 6347.7 & 791.8 \\
\hline 15 & 826.4 & 346.7 & 833.1 & 6348.2 & 1035.0 \\
\hline 20 & 1101.7 & 462.2 & 1111.0 & 6349.2 & 1442.4 \\
\hline 25 & 1377.0 & 577.7 & 1388.4 & 6353.2 & 2688.2 \\
\hline 30 & 1652.2 & 693.2 & 1666.1 & 6353.2 & 2710.3 \\
\hline \hline
\end{tabular}

DMs. If one solution must be identified to be implemented in practice, the DMs' subjective preferences should be taken into account. For example, Table III shows that the major contributor to the increase of the nominal cost is the investment cost $C_{\mathrm{INV}}$, and therefore, a DM may choose an optimal solution from the Pareto curve (see Fig. 4) that is on his/her real investment budget. More generally, along with the tradeoff curve in Fig. 4, there are some solution points for which a small cost investment gives a large gain of system resilience. Therefore, by taking a solution and its neighbor on the tradeoff curve (the less costly one), one can define a rate of change of resilience with respect to cost, $\mid \Delta p / \Delta$ cost $\mid$. This rate can be utilized as a reference to make decisions: the larger the ratio, the more preferred the solution is. In addition, the results show that increasing the number of repair crews is also able to enhance system resilience against disruptions, which is expected. However, the marginal gain in resilience generally decreases as the number of repair crews increases. Therefore, a tradeoff between the marginal cost of deploying repair crews and the marginal gain in resilience is necessary and should be carefully analyzed for rational decision making.

Additionally, this paper focuses mainly on proposing a novel quantitative decision-supporting framework that incorporates resilience requirements into the system planning/design process. The scenarios of SLD events are generated by a random mechanism, for the purpose of simplicity. The application of the framework in the real-world setting requires an attentive definition of the disruption scenarios. In fact, generating a set of relevant disruption scenarios is a part of the $\mathrm{CI}$ identification process (e.g., the EC Directive 2008/114/EC requires to develop relevant failure scenarios of a CI in order to assess the transboundary impacts on other Member States), which has attracted much attention [70]-[72]. In practice, methods like the hierarchical ontology [71] could be used to identify different types of SLD events faced by a CI system. For each SLD type, sophisticated models (e.g., for earthquakes [56] and storms [57]) can be used to generate a subset of relevant disruption scenarios, e.g., the most likely and the worst-case scenarios, by examining what the potential threats to the CI systems are and how the system components are affected. If the number of disruption scenarios is too large, a subset of the scenarios can be considered using methods like the sample average approximation [73].
Finally, the proposed system operation model does not consider detailed short-term technical constraints, like unit commitment particularly for power systems, but rather relies on an average system representation and a generic network flow model. This is because we aim to propose a scalable modeling framework that can be used for different types of CIs. Thus, the generic network flow model [3], [4], [28] is used. For its application to specific CI systems, additional physical/regulatory constraints can be carefully integrated into the operational constraints (8)-(15) and (23), (24) if necessary. Also, it would be interesting to relax other model assumptions in our future works. For instance, instead of assuming that protected facilities are invulnerable, a stochastic model may be developed to describe the impact of protection measures, e.g., describing how the failure probability of a component is reduced under different protection measures and their strengths. This requires more detailed models of specific protection measures and of the disruption events, and the development of the stochastic impact model itself.

\section{CONCLUSION}

In this paper, we presented a $p$-robust optimization model for CI network planning against SLDs. The objective of the model was to minimize the investment costs and total system costs under nominal operation conditions, which embrace economic, environmental, and societal dimensions of system operations. The resilience of the system was integrated into the planning process by forcing the $p$-robust constraints, i.e., the solution must have a relative regret of no more than $p$ in each disruption scenario considered. Importantly, the postdisruption restoration process was optimized and taken into account for the quantification of system resilience loss, different from previous perspectives that only consider the mitigation of system vulnerability, i.e., protect the system from immediate performance drop after a disruptive event. We adopted a hybrid SBD and VNS approach to solve the model, and computational experiments show that we can obtain optimal or suboptimal solutions in a fraction of the time required by CPLEX.

From a managerial point of view, we showed that system resilience can be improved greatly without substantial increases in investment, i.e., creating financial constraints to utilities with 
careful up-front planning for CIP and expansion. A tradeoff curve generated by choosing different values of parameter $p$ can help DMs choose the desired resilience level based on budget constraints. Furthermore, the results also showed that increasing the number of repair crews, within a certain region, is also an effective defense strategy to mitigate system resilience loss under SLDs. A cost-benefit analysis of increasing repair crew could be carried out to help manager choose the optimum number of repair crews.

\section{ACKNOWLEDGMENT}

The authors sincerely thank the editors and the anonymous reviewers for their invaluable comments and suggestions for improving this paper.

\section{REFERENCES}

[1] W. Kröger and E. Zio, Vulnerable Systems. New York, NY, USA: Springer, 2011.

[2] S. Hosseini and K. Barker, "Modeling infrastructure resilience using Bayesian networks: A case study of inland waterway ports," Comput. Ind. Eng., vol. 93, pp. 252-266, 2016.

[3] M. Ouyang, "Review on modeling and simulation of interdependent critical infrastructure systems," Rel. Eng. Syst. Saf., vol. 121, pp. 43-60, 2014.

[4] E. Zio, "Challenges in the vulnerability and risk analysis of critical infrastructures," Rel. Eng. Syst. Saf., vol. 152, pp. 137-150, 2016.

[5] Y. Fang and E. Zio, "Game-theoretic decision making for the resilience of interdependent infrastructures exposed to disruptions," in Critical Infrastructure Security and Resilience. New York, NY, USA: Springer, 2019, pp. $97-114$.

[6] S. Hosseini, K. Barker, and J. E. Ramirez-Marquez, "A review of definitions and measures of system resilience," Rel. Eng. Syst. Saf., vol. 145, pp. 47-61, 2016.

[7] M. Ouyang and Y. Fang, "A mathematical framework to optimize critical infrastructure resilience against intentional attacks," Comput. Aided Civil Infrastructure Eng., vol. 32, pp. 909-929, 2017.

[8] R. Westrum, "A typology of resilience situations," in Resilience Engineering. Boca Raton, FL, USA: CRC Press, 2017, pp. 67-78.

[9] Y.-P. Fang, N. Pedroni, and E. Zio, "Resilience-based component importance measures for critical infrastructure network systems," IEEE Trans. Rel., vol. 65, no. 2, pp. 502-512, Jun. 2016.

[10] Y. Fang and G. Sansavini, "Optimum postdisruption restoration for enhanced infrastructure network resilience: A fuzzy programming approach," in Proc. Eur. Saf. Rel. Conf., Glasgow, U.K., 2016, pp. 587-594.

[11] G. Brown et al., "Defending critical infrastructure," Interfaces, vol. 36, no. 6 , pp. 530-544, 2006.

[12] C. Zhang and J. E. Ramirez-Marquez, "Protecting critical infrastructures against intentional attacks: A two-stage game with incomplete information," IIE Trans., vol. 45, no. 3, pp. 244-258, 2013.

[13] C. Zhang, J. E. Ramirez-Marquez, and J. Wang, "Critical infrastructure protection using secrecy-A discrete simultaneous game," Eur. J. Oper. Res., vol. 242, no. 1, pp. 212-221, 2015.

[14] B. C. Ezell, J. V. Farr, and I. Wiese, "Infrastructure risk analysis model," J. Infrastructure Syst., vol. 6, no. 3, pp. 114-117, 2000.

[15] E. E. Lee, J. E. Mitchell, and W. A. Wallace, "Assessing vulnerability of proposed designs for interdependent infrastructure systems," in Proc. 37th Annu. Hawaii Int. Conf. Syst. Sci., 2004, p. 8.

[16] S. Bao et al., "An integrated tri-level model for enhancing the resilience of facilities against intentional attacks," Ann. Oper. Res., pp. 1-31, 2017.

[17] M. Ouyang, "A mathematical framework to optimize resilience of interdependent critical infrastructure systems under spatially localized attacks," Eur. J. Oper. Res., vol. 262, no. 3, pp. 1072-1084, 2017.

[18] Y. Fang and G. Sansavini, "Optimizing power system investments and resilience against attacks," Rel. Eng. Syst. Saf., vol. 159, pp. 161-173, 2017.

[19] M. Ouyang et al., "Mitigating electric power system vulnerability to worstcase spatially localized attacks," Rel. Eng. Syst. Saf., vol. 165, pp. 144-154, 2017.

[20] Y. Fang, N. Pedroni, and E. Zio, "Optimization of cascade-resilient electrical infrastructures and its validation by power flow modeling," Risk Anal. vol. 35, no. 4, pp. 594-607, 2015.
[21] C. Zhang, J. E. Ramirez-Marquez, and Q. Li, "Locating and protecting facilities from intentional attacks using secrecy," Rel. Eng. Syst. Saf., vol. 169, pp. 51-62, 2018.

[22] M. Bruneau et al., "A framework to quantitatively assess and enhance the seismic resilience of communities," Earthquake Spectra, vol. 19, no. 4, pp. 733-752, 2003.

[23] Z. Çagnan and R. Davidson, "Postearthquake restoration modeling of electric power systems," in Proc. 13th World Conf. Earthquake Eng., 2004, pp. 1-6.

[24] V. Ramachandran et al., "Framework for modeling urban restoration resilience time in the aftermath of an extreme event," Natural Hazards Rev., vol. 16, no. 4, 2015, Art. no. 04015005.

[25] C. Lian and Y. Y. Haimes, "Managing the risk of terrorism to interdependent infrastructure systems through the dynamic inoperability inputoutput model," Syst. Eng., vol. 9, no. 3, pp. 241-258, 2006.

[26] Y. Y. Haimes et al., "Inoperability input-output model for interdependent infrastructure sectors. I: Theory and methodology," J. Infrastructure Syst., vol. 11, no. 2, pp. 67-79, 2005.

[27] S. Argyroudis et al., "Systemic seismic risk assessment of road networks considering interactions with the built environment," Comput. Aided Civil Infrastructure Eng., vol. 30, no. 7, pp. 524-540, 2015.

[28] S. G. Nurre et al., "Restoring infrastructure systems: An integrated network design and scheduling (INDS) problem," Eur. J. Oper. Res., vol. 223, no. 3, pp. 794-806, 2012.

[29] C. Coffrin, P. Van Hentenryck, and R. Bent, "Last-mile restoration for multiple interdependent infrastructures," in Proc. AAAI Conf. Artif. Intell., 2012, pp. 455-463.

[30] A. D. González et al., "The interdependent network design problem for optimal infrastructure system restoration," Comput. Aided Civil Infrastructure Eng., vol. 31, pp. 334-350, 2015.

[31] E. E. Lee II, J. E. Mitchell, and W. A. Wallace, "Restoration of services in interdependent infrastructure systems: A network flows approach," IEEE Trans. Syst., Man, Cybern. C, Appl. Rev., vol. 37, no. 6, pp. 1303-1317, Nov. 2007.

[32] E. Miller-Hooks, X. Zhang, and R. Faturechi, "Measuring and maximizing resilience of freight transportation networks," Comput. Oper. Res., vol. 39, no. 7, pp. 1633-1643, 2012.

[33] A. Deshmukh and M. Hastak, "Enhancing post disaster recovery by optimal infrastructure capacity building," Int. J. Res. Eng. Technol., vol. 3, pp. 5-12, 2014.

[34] C. Losada, M. P. Scaparra, and J. R. O'Hanley, "Optimizing system resilience: A facility protection model with recovery time," Eur. J. Oper. Res., vol. 217, no. 3, pp. 519-530, 2012.

[35] S. E. Chang, "Evaluating disaster mitigations: methodology for urban infrastructure systems," Natural Hazards Rev., vol. 4, no. 4, pp. 186-196, 2003.

[36] P. Hansen and N. Mladenović, "Variable neighborhood search: Principles and applications," Eur. J. Oper. Res., vol. 130, no. 3, pp. 449-467, 2001.

[37] C.-H. Yang et al., "Optimal robust ordering quantity for a new product under environmental constraints," IEEE Trans. Eng. Manage., to be published, doi: 10.1109/TEM.2018.2819192.

[38] D. L. Alderson, G. G. Brown, and W. M. Carlyle, "Assessing and improving operational resilience of critical infrastructures and other systems," INFORMS Tut. Oper. Res.: Bridging Data Decis., pp. 180-215, 2014.

[39] D. L. Alderson, G. G. Brown, and W. M. Carlyle, "Operational models of infrastructure resilience," Risk Anal., vol. 35, no. 4, pp. 562-586, 2015.

[40] P. Vassilopoulos, "Models for the identification of market power in wholesale electricity markets," UFR Sci. Org., Paris, France, DEA 129, 2003.

[41] A. Arab et al., "Stochastic prehurricane restoration planning for electric power systems infrastructure," IEEE Trans. Smart Grid, vol. 6, no. 2, pp. 1046-1054, Mar. 2015.

[42] Study on the Estimation of the Cost of Disruption of Gas Supply in Europe, ACER, New Taipei City, Taiwan, 2018.

[43] S. Buck et al., "Measuring welfare losses from urban water supply disruptions," J. Assoc. Environ. Resour. Econ., vol. 3, no. 3, pp. 743-778, 2016.

[44] L. V. Snyder and M. S. Daskin, "Stochastic p-robust location problems," IIE Trans., vol. 38, no. 11, pp. 971-985, 2006.

[45] P. Peng et al., "Reliable logistics networks design with facility disruptions," Transp. Res. Part B: Methodol., vol. 45, no. 8, pp. 1190-1211, 2011.

[46] G. J. Gutiérrez, P. Kouvelis, and A. A. Kurawarwala, "A robustness approach to uncapacitated network design problems," Eur. J. Oper. Res., vol. 94, no. 2, pp. 362-376, 1996.

[47] D. Van Hertem et al., Usefulness of DC power flow for active power flow analysis with flow controlling devices," in Proc. 8th IEE Int. Conf. AC DC Power Transmiss., 2006, pp. 58-62. 
[48] R. Hemmati, R.-A. Hooshmand, and A. Khodabakhshian, "Comprehensive review of generation and transmission expansion planning," IET Gener., Transmiss. Distrib., vol. 7, no. 9, pp. 955-964, 2013.

[49] Y. Fang and G. Sansavini, "Emergence of antifragility by optimum postdisruption restoration planning of infrastructure networks," J. Infrastructure Syst., vol. 23, no. 4, 2017, Art. no. 04017024.

[50] P. Bonami, M. Kilinç, and J. Linderoth, "Algorithms and software for convex mixed integer nonlinear programs," in Mixed Integer Nonlinear Programming. New York, NY, USA: Springer, 2012, pp. 1-39.

[51] S. Dempe, Foundations of Bilevel Programming. New York, NY, USA: Springer, 2002.

[52] P. Hansen, B. Jaumard, and G. Savard, "New branch-and-bound rules for linear bilevel programming," SIAM J. Sci. Statist. Comput., vol. 13, no. 5, pp. 1194-1217, 1992.

[53] A. Shapiro and A. Nemirovski, "On complexity of stochastic programming problems," in Continuous Optimization. New York, NY, USA: Springer, 2005, pp. 111-146.

[54] M. Ouyang, "Critical location identification and vulnerability analysis of interdependent infrastructure systems under spatially localized attacks," Rel. Eng. Syst. Saf., vol. 154, pp. 106-116, 2016.

[55] Y. Berezin et al., "Localized attacks on spatially embedded networks with dependencies," Sci. Rep., vol. 5, 2015, Art. no. 8934.

[56] B.-J. Chiou and R. R. Youngs, "An NGA model for the average horizontal component of peak ground motion and response spectra," Earthquake Spectra, vol. 24, no. 1, pp. 173-215, 2008.

[57] H. Liu, R. A. Davidson, and T. V. Apanasovich, "Spatial generalized linear mixed models of electric power outages due to hurricanes and ice storms," Rel. Eng. Syst. Saf., vol. 93, no. 6, pp. 897-912, 2008.

[58] J. Salmeron, K. Wood, and R. Baldick, "Worst-case interdiction analysis of large-scale electric power grids," IEEE Trans. Power Syst., vol. 24, no. 1, pp. 96-104, Feb. 2009.

[59] E. Yamangil, "Valid inequalities for mixed-integer linear programming problems," doctoral dissertation, Graduate School-New Brunswick, Rutgers Univ., New Brunswick, NJ, USA, 2015.

[60] J. Lazić et al., "Variable neighbourhood decomposition search for 0-1 mixed integer programs," Comput. Oper. Res., vol. 37, no. 6, pp. 1055$1067,2010$.

[61] P. Hansen, C. Oğuz, and N. Mladenović, "Variable neighborhood search for minimum cost berth allocation," Eur. J. Oper. Res., vol. 191, no. 3, pp. 636-649, 2008.

[62] V. C. Hemmelmayr, K. F. Doerner, and R. F. Hartl, "A variable neighborhood search heuristic for periodic routing problems," Eur. J. Oper. Res., vol. 195, no. 3, pp. 791-802, 2009.

[63] W. Bukhsh and K. McKinnon, Network Data of Real Transmission Networks, 2013. [Online]. Available: http://www.maths.ed.ac.uk/ optenergy/NetworkData

[64] C. Ruiz and A. J. Conejo, "Robust transmission expansion planning," Eur. J. Oper. Res., vol. 242, no. 2, pp. 390-401, 2015.

[65] D. Louth, Governor's Two-Storm Panel: Distribution Infrastructure Hardening Options and Recommendations. Berlin, CT, USA: Connecticut Light \& Power, 2011.

[66] K. W. Bell and A. N. D. Tleis, "Test system requirements for modelling future power systems," in Proc. IEEE Power Energy Soc. Gen. Meeting, 2010, pp. 1-8.

[67] C. Grigg, "The IEEE Reliability Test System-1996. A report prepared by the reliability test system task force of the application of probability methods subcommittee," IEEE Trans. Power Syst., vol. 14, no. 3, pp. 10101020, Aug. 1999.

[68] J. Salmeron, K. Wood, and R. Baldick, "Analysis of electric grid security under terrorist threat," IEEE Trans. Power Syst., vol. 19, no. 2, pp. 905912, May 2004.

[69] I. Schlecht and H. Weigt, Swissmod- A model of the Swiss electricity market. Center of Business and Economics (WWZ), University of Basel, Peter Merian-Weg 6, 4002 Basle, Switzerland, Tech. Rep. Apr. 2014

[70] W. Klibi and A. Martel, "Scenario-based supply chain network risk modeling," Eur. J. Oper. Res., vol. 223, no. 3, pp. 644-658, 2012.

[71] P. Trucco, B. Petrenj, and M. De Ambroggi, "Ontology-based disruption scenario generation for critical infrastructure," in Proc. Probab. Saf. Assessment Manage. Conf., 2014, pp. 1-11.

[72] N. Komendantova et al., "Multi-hazard and multi-risk decision-support tools as a part of participatory risk governance: Feedback from civil protection stakeholders," Int. J. Disaster Risk Reduction, vol. 8, pp. 50-67, 2014.

[73] A. J. Kleywegt, A. Shapiro, and T. Homem-de-Mello, "The sample average approximation method for stochastic discrete optimization," SIAM J. Optim., vol. 12, no. 2, pp. 479-502, 2002.

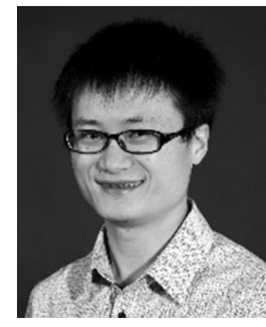

Yi-Ping Fang (M'17) received the Ph.D. degree in industrial engineering from École Centrale Paris, Gif-sur-Yvette, France, in 2015.

$\mathrm{He}$ is currently an Assistant Professor with the Chair on Systems Science and the Energy Challenge, Fondation Electricité de France, with a joint appointment with the École CentraleSupélec, Université Paris-Saclay, Gif-sur-Yvette. His research interests include the study and development of advanced computational methods for risk, reliability, and resilience analysis of critical infrastructures.

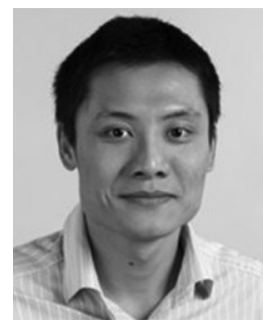

Chao Fang received the B.S. degree in information engineering and M.S. degree in systems engineering from Xi' an Jiaotong University, Xi' an, China, in 2005 and 2008, respectively, and the Ph.D. degree in industrial engineering from École Centrale Paris, Gif-surYvette, France in 2011.

$\mathrm{He}$ is currently an Associate Professor in risk management and engineering management with the School of Economics and Management, Wuhan University, Wuhan, China. His research interests include system modeling and simulation. project management, risk management, and complex

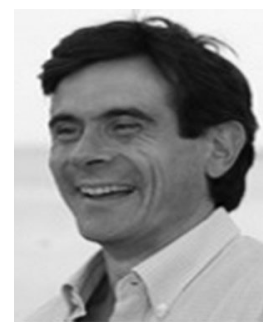

Enrico Zio (SM'09) received the B.S. and Ph.D. degrees in nuclear engineering from the Politecnico di Milano, Milano, Italy, in 1991 and 1995, respectively, the M.Sc. degree in mechanical engineering from the University of California, Los Angeles, CA, USA, in 1995, and the Ph.D. degree in nuclear engineering from the Massachusetts Institute of Technology, Cambridge, MA, USA, in 1998.

He is currently a Full Professor in risk and reliability with the Centre for Research on Risk and Crisis, Ecole de Mines, ParisTech, PSL Research University, Sophia Antipolis, France. He is also a Full Professor with the Politecnico di Milano. He has authored or coauthored seven books and more than 300 papers in international journals. His research interests include the modeling and analysis of complex systems' reliability, maintainability, prognostics, safety, vulnerability, resilience, and security characteristics.

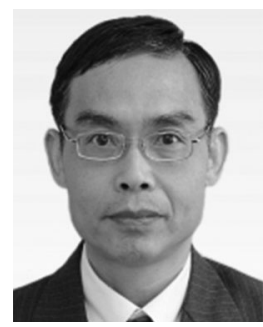

Min Xie (F'06) received the Ph.D. degree in quality technology from Linköping University, Linköping, Sweden, in 1987.

He is currently a Chair Professor with the Department of Systems Engineering and Engineering Management, City University of Hong Kong, Hong Kong. He has authored or coauthored more than 200 journal articles and eight books. His research interests include statistical quality and reliability engineering. 